\title{
Avaliação do Conhecimento de Alunos do Internato Médico sobre Pancreatite Aguda Utilizando a Aprendizagem Baseada em Problemas
}

\section{Evaluating Medical Interns' Knowledge about Acute Pancreatitis Using Problem-Based Learning}

Leonardo Castro Marinzeck ${ }^{I}$

Eduardo Stefaneli

Danilo Cesar Paschoalino ${ }^{I}$

Edilson Carlos Caritá ${ }^{I}$

Silvia Sidneia da Silva ${ }^{I}$

\section{PALAVRAS-CHAVE}

- Pancreatite.

- Internato e Residência.

- Aprendizagem Baseada em Problemas (ABP)

\section{RESUMO}

A pancreatite aguda é doença frequente nas enfermarias de hospitais gerais, tem etiologia multifatorial e pode levar a alterações graves dos diversos aparelhos e sistemas corpóreos. O internato, em geral, é o momento em que os alunos de Medicina sedimentam os conhecimentos adquiridos de maneira fracionada durante as etapas básicas do curso. Atualmente, com a mudança do modelo pedagógico em vários cursos de Medicina, a Aprendizagem Baseada em Problemas (ABP) vem sendo adotada. $O$ objetivo deste estudo foi avaliar a efetividade do método $A B P$ na aquisição de conhecimentos de alunos do internato médico sobre pancreatite aguda, em comparação ao método tradicional. Trata-se de estudo descritivo, de abordagem quantitativa e caráter transversal. A amostra foi composta por 40 alunos que cursavam a décima primeira etapa do curso de Medicina de uma instituição de ensino superior privada do interior paulista no segundo semestre de 2015. Foi realizada avaliação entre os sujeitos do grupo pesquisa e do grupo controle quanto à diferença no nível de conhecimento sobre o tema. Como teste estatístico foi utilizado o teste paramétrico $t$ de Student para dois grupos independentes. Como resultado, obteve-se que os alunos que participaram das sessões tutoriais pelo método ABP tiveram maior rendimento nas respostas às questões do instrumento de avaliação, com $p=0,013$. Assim, ratifica-se que, além dos ganhos já sabidos em relação à formação de um profissional médico proativo, atento para sentir seu ambiente de trabalho, seus pares e os usuários, o método é também eficiente para a continuidade do processo ensino-aprendizagem durante o internato médico. Desse modo, recomenda-se utilizar a ABP no processo ensino-aprendizagem da Clínica Cirúrgica na universidade do estudo. Propõe-se também a continuidade de pesquisas com médicos residentes para avaliar a aquisição do conhecimento de outras patologias em outras clínicas, comparando-se os métodos ABP e o tradicional com vistas a mais subsídios para adoção da ABP nesse estágio de formação. 


\section{KEY-WORDS}

- Pancreatitis.

- Internship and Residency.

- Problem Based Learning (PBL).

\begin{abstract}
Acute pancreatitis is a frequent disease in general hospitals wards; it has a multifactorial etiology and can lead to severe alterations of the various corporeal systems. The internship generally represents the time when medical students consolidate the knowledge they have gradually acquired during the initial stages of their course. Several medical courses are currently undergoing a change in pedagogical focus, with the adoption of Problem-Based Learning (PBL). The objective of this study was to evaluate the effectiveness of the Problem-Based Learning (PBL) method in relation to knowledge about acute pancreatitis among medical intern students from a private university in the interior of São Paulo state, compared to the traditional learning method. It is a descriptive and transversal study with a quantitative approach. The sample consisted of 40 sixth-year medicine students of a private higher education institution in São Paulo state, in the second semester of 2015. A comparative evaluation was made between the knowledge about the matter among the study group and the control group. The Student $t$ parametric test was applied to two independent groups, resulting in the finding that the students who participated in the PBL tutorial sessions performed better in the assessment test, with $p=0.013$. Hence, in addition to the advantages already known in relation to the preparation of proactive medical professionals, in tune with the feelings of their working environment, their peers and patients, the method is also proven to be efficient in continuing the teaching-learning process during medical internship. It is therefore concluded that this teaching-learning method be employed at the medical school in the study. It is also proposed that the research be continued with resident physicians, to evaluate the acquisition of knowledge of other pathologies and other specialties, comparing PBL to traditional methods in order to achieve greater support for the adoption of the PBL method in this stage of medical training.
\end{abstract}

Recebido em: 19/9/2018

Aceito em: 25/9/2018

\section{INTRODUÇÃO}

Com a mudança do modelo pedagógico em vários cursos de Medicina, no Brasil e no mundo, o método da Aprendizagem Baseada em Problemas (ABP) vem ganhando espaço em várias instituições de ensino. Trata-se de metodologia que visa à formação de um profissional indagador e que tem como alicerce da sua formação a visão humanística e de inter-relação com os usuários e demais profissionais que o circundam ${ }^{1}$.

A formação clássica nos cursos de Medicina em que o professor, baseado em sua cátedra, transmite de maneira unidirecional seus conhecimentos e análises críticas ao alunado molda um profissional médico sem a perspicácia de indagar, de se relacionar e de questionar as formas como esse conhecimento foi estabelecido. Com o estímulo utilizado na ABP, prioriza-se a construção do próprio conhecimento, e a maneira engessada de transferência, até então usada, torna-se dinâmica, permitindo que fatores externos, intimamente ligados ao processo saúde-doença, sejam absorvidos e incorporados na prática clínica diária do novo profissional ${ }^{2}$.

Segundo Feuerwerker ${ }^{3}$, desde o início do século XXI, as escolas médicas brasileiras encontram-se diante do desafio de mudar para formar um profissional crítico, capaz de aprender a aprender, de trabalhar em equipe, de considerar a realidade social para prestar atenção humana e de qualidade. As mudanças necessárias são profundas porque implicam a transformação de concepções, práticas e relações de poder tanto nos espaços internos das universidades, como em suas relações com a sociedade, especialmente com os serviços de saúde e a população.

No internato médico, período em que os acadêmicos do curso de Medicina mantêm maior contato com os usuários, a ABP busca um modo mais ativo de consolidar o conhecimento adquirido anteriormente, por meio do intenso exercício da prática médica ${ }^{4}$, incluindo a revisitação aos conteúdos discutidos sobre pancreatite aguda.

A pancreatite aguda tem apresentação clínica e gravidade muito variáveis ${ }^{5}$. Para alguns autores ${ }^{6}$, a forma grave representa uma pequena percentagem das admissões em Unidades de Terapia Intensiva (UTI) polivalentes, mas está associada a um significativo consumo de recursos humanos e técnicos, além de apresentar elevada taxa de mortalidade e morbidade nas UTI e nos hospitais gerais, por envolver, geralmente, longos tempos de internação. 
Como a $\mathrm{ABP}$ vem sendo adotada em muitos cursos de graduação em Medicina, este estudo buscou estabelecer se há aquisição de conhecimento no internato médico, fase em que os estudantes já passaram pelas etapas básicas e devem sedimentar o conhecimento adquirido nesse processo. Desse modo, refletiremos sobre o uso e os benefícios da ABP no término da formação de médicos no internato, considerando a pancreatite aguda como tema a ser explorado.

Nessa direção, o objetivo do estudo foi avaliar a efetividade do método ABP na aquisição de conhecimentos sobre pancreatite aguda por alunos do internato médico de uma universidade privada em comparação ao método tradicional.

\section{METODOLOGIA}

Trata-se de estudo descritivo, de abordagem quantitativa e caráter transversal que avaliou o conhecimento dos alunos sobre o tema selecionado, considerando a aplicação do método de ensino tradicional e o método ABP no processo de ensino-aprendizagem no final do estágio de Clínica Cirúrgica. Pela característica quantitativo-descritiva, utilizou como técnica a aplicação de um instrumento de avaliação para quantificar se houve ganho no conhecimento do grupo estudado sobre o grupo controle.

A pesquisa foi realizada em Ribeirão Preto, cidade do Nordeste paulista, com alunos do curso de Medicina de uma instituição de ensino superior privada. A população da presente investigação constituiu-se da totalidade dos alunos matriculados na décima primeira etapa (58 estudantes) do segundo semestre do ano de 2015.

Como critérios de inclusão para participação na pesquisa, o estudante deveria estar matriculado na décima primeira etapa do curso de Medicina da instituição em questão, concordar em assinar o Termo de Consentimento Livre e Esclarecido após ciência dos objetivos da investigação e responder ao instrumento de avaliação em no máximo dez dias após o término das atividades de aprendizagem. Depois da aplicação dos critérios de inclusão, a amostra constituiu-se de 40 indivíduos.

$\mathrm{O}$ instrumento de coleta de dados foi um questionário com 30 questões sobre o tema específico, validado por 11 especialistas das áreas de Cirurgia Geral, Cirurgia do Aparelho Digestivo, Gastrenterologia e por cinco médicos egressos da Universidade de Ribeirão Preto (Unaerp), atendo-se ao cuidado de ouvir os especialistas e também os egressos do internato médico, considerando a relevância das questões propostas, bem como o entendimento delas pelos residentes da área. Para a elaboração desse instrumento foram consultados profissionais que lidam em seu cotidiano com a doença pesquisada e que têm íntimo contato com o ensino, além de serem, no mínimo, especialistas da área cirúrgica ou clínica da Gastrente- rologia. As questões sobre o tema específico visam avaliar o conhecimento do médico generalista sem incorrer na possibilidade de supervalorizar conhecimento pormenorizado.

As sessões tutoriais, a aula teórica e os testes ocorreram nos meses de agosto e setembro de 2015, período de realização do estágio em Cirurgia Geral da décima primeira turma do curso de Medicina da instituição estudada.

Inicialmente, foi ministrada a aula do tema específico utilizando-se no processo ensino-aprendizagem o método tradicional para os alunos do grupo controle. Na semana seguinte, foram realizadas as aberturas das tutorias com os alunos participantes do grupo ABP. Tanto a aula teórica como as sessões tutoriais foram ministradas pelo próprio pesquisador, professor do curso de Medicina da referida instituição.

Dez dias após ser ministrada a aula teórica e concluídas as sessões tutoriais, foi aplicado o instrumento de avaliação aos 40 alunos participantes, sendo 19 do grupo controle e 21 do grupo ABP. Após a aplicação do questionário, as questões foram corrigidas, e as notas absolutas agrupadas em intervalo de classe definido a critério do pesquisador.

As notas de cada grupo foram comparadas estatisticamente a fim de analisar se houve significância na aquisição de conhecimento sobre pancreatite aguda no grupo submetido à sessão tutorial (grupo pesquisa) em relação ao grupo controle.

A análise estatística realizada identificou e comparou as notas dos alunos submetidos à sessão tutorial e as notas dos alunos do grupo controle. Como teste estatístico foi utilizado o teste paramétrico $t$ de Student para dois grupos independentes, com as seguintes equações:

$$
\mathrm{T}_{0}=\frac{\text { média grupo } \mathrm{ABP}-\text { média grupo controle }}{\text { desvio }- \text { padrão combinado } \cdot \sqrt{\left(\frac{1}{\mathrm{~N} 1}+\frac{1}{\mathrm{~N} 2}\right)}}
$$

Desvio - padrão combinado $=\sqrt{\frac{(\mathrm{N} 1-1) \cdot(\mathrm{Var} \cdot \mathrm{G} 1)^{2}+(\mathrm{N} 2-1) \cdot(\mathrm{Var} \cdot \mathrm{G} 2)^{2}}{\text { grau de liberdade } \mathrm{N} 1+\mathrm{N} 2-2}}$

O estudo foi apreciado e autorizado pela coordenação geral de graduação e pelo coordenador do curso de graduação em Medicina da instituição estudada, bem como autorizado pelo Comitê de Ética em Pesquisa com Seres Humanos da Universidade de Ribeirão Preto, sob Parecer nº 1.246.926.

\section{RESULTADOS}

O perfil dos alunos do sexto ano do curso médico que compuseram a amostra do estudo, em números absolutos, constituiu-se de 22 estudantes do sexo feminino e 18 do masculino. A média de idade foi de 25,627 anos, com desvio-padrão de 3,140 anos e mediana de 25 anos. 
O Gráfico 1 demonstra a distribuição dos 19 alunos do grupo controle, subdivididos em variações das notas obtidas da menor para a maior, comprovando que a distribuição dos resultados segue um padrão normal da curva de Gauss.

No Gráfico 2 está a distribuição dos 21 alunos do grupo ABP, subdivididos em variações das notas obtidas da menor para a maior, comprovando que a distribuição dos resultados também segue um padrão normal, formando uma curva gaussiana.
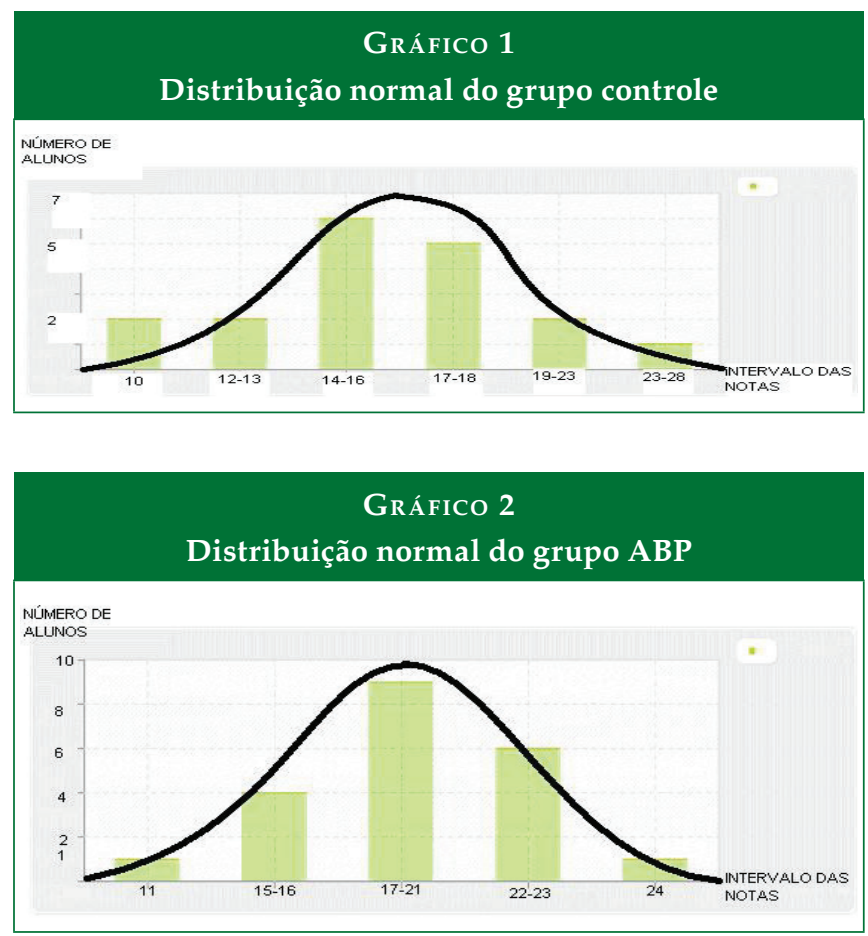

Para a análise dos dados do grupo ABP (Tabela 1), considerou-se que n é o número de participantes do grupo (21 estudantes), o somatório dos dados é a soma das notas obtidas por todos os participantes do grupo n, e a média aritmética é a divisão da soma dos dados por $n$. O desvio-padrão é uma medida de dispersão, normalmente associada com a média, e afere a variabilidade dos valores à volta da média, com a variância sendo o desvio-padrão ao quadrado, significando o segundo momento de dispersão.

\begin{tabular}{|lc|}
\multicolumn{2}{c}{$\begin{array}{c}\text { TABELA 1 } \\
\text { Estatística descritiva do grupo ABP }\end{array}$} \\
\hline Indicadores & Resultados \\
\hline $\mathrm{n}$ & 21 \\
\hline Somatório dos dados & 408 \\
\hline Média aritmética & 19,43 \\
\hline Mediana & 20 \\
\hline Desvio-padrão & 3,34 \\
\hline Variância & 11,16 \\
\hline
\end{tabular}

A análise dos dados relativa ao grupo de controle (Tabela 2), que utilizou o processo ensino-aprendizagem pela metodologia tradicional, incluiu 19 estudantes e obedece ao delineamento utilizado com o grupo ABP.

\begin{tabular}{|lc|}
\multicolumn{2}{c}{ TABELA 2} \\
Estatística descritiva do grupo controle \\
\hline Indicadores & Resultados \\
\hline $\mathrm{n}$ & 19 \\
\hline Somatório dos dados & 315 \\
\hline Média aritmética & 16,58 \\
\hline Mediana & 16 \\
\hline Desvio-padrão & 4,45 \\
\hline Variância & 19,81
\end{tabular}

Para determinar os valores finais deste estudo, utilizou-se o teste de Intervalo de Confiança para a Diferença entre Duas Médias em pequenas amostras. Porém, por haver dois grupos em estudo e assumindo-se que os desvios-padrão populacionais são comuns a ambos os grupos, combinou-se os desvios-padrão amostrais para formar uma estimativa combinada, denominada Desvio-Padrão Combinado (DPC), cujo valor encontrado foi 15,25 . A partir do cálculo deste, pode-se chegar a $\mathrm{T}_{0}=0,595$.

A comparação das médias dos dois grupos foi realizada pelo teste $\mathrm{t}$ de Student, considerando o nível de significância de 0,05 . Nesse caso, acredita-se que a média do número de acertos tem uma distribuição normal.

Como o t crítico pela tabela t Student, com $\mathrm{p}=0,05$, é 1,6840 , pode-se rejeitar a hipótese $H_{0}$ (pois sua rejeição deve ser considerada quando ( $\mathrm{p}$-valor $\leq \mathrm{T}_{0} \leq+\mathrm{p}$-valor), ou seja, $-1,6840 \leq 0,595 \leq+1,6840$ ) e aceitar a hipótese $H_{1}$. Assim, o teste mostra que existe significância em relação ao ganho de conhecimento do grupo ABP sobre o grupo controle.

O grupo controle teve média de 16,57 acertos, com desvio-padrão de 4,5, enquanto o grupo ABP teve média de 19,42 e desvio-padrão de 3,3. O teste $t$ de Student detectou diferença ( $p=0,013$ ) em favor do grupo ABP, mostrando, portanto, que o método $\mathrm{ABP}$ foi mais eficiente que o método tradicional na amostra estudada.

No caso de contestação de que a média dos acertos não tem distribuição normal, provas de Wilcoxon e de Mann-Whitney foram usadas como testes não paramétricos ${ }^{7}$ alternativos, que comparam a média dos pontos e, em muitos casos, a mediana. Encontrou-se, para o grupo controle, uma média de pontos de 15,8, e para o grupo ABP, 24,7. O valor de $\mathrm{p}$ foi de 0,016. Assim, como no teste t de Student, evidencia-se que, no presente estudo, o método ABP foi mais eficiente que o método tradicional em relação ao tema específico. 


\section{DISCUSSÃO}

A principal preocupação quanto ao método $\mathrm{ABP}$ se pauta no perfil do egresso, que sabidamente, consegue, de maneira ativa, buscar o conhecimento teórico, além da nobre premissa de ter em sua formação o alicerce para lidar com as várias nuances do processo saúde-doença, sendo que inúmeros personagens atuam em seus mais variados níveis de formação para a construção de um bem-estar individual e, principalmente, coletivo ${ }^{8,9}$.

Porém, os resultados de vários pesquisadores demonstram a dificuldade, por parte dos professores e também dos estudantes, de continuar o processo de aprendizagem ativo no internato médico na perspectiva da $\mathrm{ABP}$ voltada aos estudantes e pacientes ${ }^{10}$.

Tais estudos trazem alguns questionamentos que inferem inconsistências na metodologia, tais como: alunos individualistas, competitivos e introvertidos têm dificuldade de adaptação à metodologia ABP; o aprofundamento nos assuntos compromete o tempo que poderia ser dedicado a maior abrangência de conteúdos; os docentes podem sentir desconforto psíquico decorrente da tendência do método ABP de testar a flexibilidade e o conhecimento do professor; os docentes se queixam de que o trabalho em grupo dificulta a avaliação individual, entre outros ${ }^{11}$.

Alguns estudos mostram que, aparentemente, os alunos formados por esse método não apresentam vantagens sobre os estudantes de métodos tradicionais nas provas e concursos realizados imediatamente após o final do curso médico, mas outras pesquisas sugerem maior retenção do conhecimento após um ano quando usada a $\mathrm{ABP}^{9}$. No presente estudo, houve maior aquisição de conhecimento no grupo $\mathrm{ABP}$ quando comparado ao tradicional, de forma imediata, constatada na avaliação aplicada durante a pesquisa.

Na instituição onde ocorreu o estudo, existe um processo contínuo de capacitação docente, e não se encontram resistências para aplicação do método, tanto pelos docentes quanto pelos acadêmicos, pois tiveram toda a sua aprendizagem anterior baseada no método ABP. Ainda assim, para cada ponto entendido como dificultador do aprendizado, temos um contraditório que evoca compensações, como, por exemplo: estimular a colaboração e o estabelecimento de parcerias entre os alunos e destes com o professor; ensinar o aluno a aprender é mais vantajoso que arriscar transmitir-lhe todos os conceitos e esperar que os incorpore à prática futura; a dinâmica interdisciplinar de trabalho com problemas - reais ou simulados - promove o sentimento de grupo entre os docentes; e estudantes são mais bem avaliados por supervisores ou empregadores por demonstrarem desenvoltura profissional, iniciativa e espírito empreendedor excelentes.

Ainda de acordo com pesquisas de Couto et al. ${ }^{12}$, professores que atuaram como facilitadores no método ABP, apro- veitando as diversas ideias e interesses na resolução de problemas pelos alunos e não apenas se limitando a estudos de casos teóricos, obtiveram resultados excelentes em se tratando de conteúdo de aprendizagem. Desse estudo participaram 252 alunos do curso de Medicina do quinto ao oitavo ano, com média de idades de 23,4 anos; 95\% deles aprovaram plenamente os procedimentos de aprendizagem, classificando o método - com um tutor especializado que domina a área de conhecimento da matéria discutida em tutorias - como muito bom na construção do conhecimento; somente 16 estudantes (6\%) concordaram similarmente quando a mesma matéria discutida em tutorias tinha um não especialista como facilitador ( $p<0,0001)$. Outros resultados confirmam esse achado pelos seguintes números, por exemplo, no critério (1) orientando o processo de aprendizagem (93\% contando com um tutor especialista e $7 \%$ sem o especialista); (2) gerando objetivos de aprendizagem de estudo autônomo (87\% e 15\%, respectivamente); (3) motivando estudo autônomo ( $80 \%$ versus $15 \%$ ); (4) alcançando a aprendizagem cognitiva (92\% versus $18 \%$, respectivamente entre os métodos); (5) preparando o teste de fim de unidade ( $76 \%$ versus $6 \%$ ).

No curso de Medicina, objeto deste estudo, o método ABP é bem consolidado, atendendo às peculiaridades da instituição, do curso e dos alunos, sem perder, nessas adaptações, a essência da metodologia, que visa à formação de um médico generalista, indagador, proativo e inserido no contexto do processo saúde-doença.

Esse tema específico foi escolhido devido ao fato de a pancreatite aguda ser uma doença de grande importância na prática clínica, definida como inflamação do pâncreas que pode levar ao envolvimento de tecidos locais ou acometimento de outros órgãos de forma sistêmica, necessitando, nesses casos, de cuidados em terapia intensiva ${ }^{13}$, o que demanda do aluno raciocínio clínico que deverá se pautar em conhecimentos sólidos.

$\mathrm{O}$ aprendizado em pancreatite aguda, principalmente pelo método $\mathrm{ABP}$, durante o internato, faz com que o aluno acesse seu conhecimento prévio sobre o tema, despertando conhecimentos essenciais relacionados à história natural da doença, cujo tratamento tem como base o bloqueio do mecanismo fisiopatológico perpetuador do processo inflamatório ${ }^{14}$.

Frente aos resultados aqui expostos, vimos que o método ABP oferece aos alunos um ganho significativo de aprendizagem em relação ao método tradicional quando analisado o conhecimento teórico específico, corroborando a análise de Paulin e Poças ${ }^{15}$ em estudo realizado na Faculdade de Medicina de Bragança Paulista, no estágio de Psiquiatria, durante o internato, onde o método ABP foi aplicado com aquisição de conhecimento nessa área específica. 


\section{CONCLUSÃO}

No presente estudo, foram comparados dois métodos de ensino-aprendizagem - o tradicional (aula expositiva) e por ABP -, considerando o conhecimento de 40 alunos do internato médico sobre pancreatite aguda, de uma instituição privada do interior paulista, identificando significância estatística em relação ao ganho de conhecimento do grupo ABP sobre o grupo controle.

Ficou evidenciado que o método $\mathrm{ABP}$, além dos ganhos já sabidos em relação à formação de um profissional proativo, atento para sentir seu ambiente de trabalho, seus pares e os usuários, é também eficiente para a continuidade do processo ensino-aprendizagem durante o internato médico.

Como este estudo foi proposto para amostra e doença específicas, recomenda-se a continuidade de pesquisas com médicos residentes para avaliar a aquisição do conhecimento de outras patologias em outras clínicas comparando-se os métodos ABP e tradicional, com vistas à obtenção de mais subsídios para adoção da ABP nesse estágio de formação.

\section{REFERÊNCIAS}

1. Husain A. Problem-based Learning: a current model of education. Oman Med J. 2011 Jul;26(4):295.

2. Mitre SM, Siqueira-Batista R, Girardi-de-Mendonça JM, Morais-Pinto NM, Meirelles CAB, Pinto-Porto C et al. Metodologias ativas de ensino-aprendizagem na formação profissional em saúde: debates atuais. Ciênc. saúde coletiva 2008; 13(Sup2):2133-44.

3. Feuerwerker LCM. Mudanças na Educação médica: os casos de Londrina e Marília. São Paulo: Faculdade de Saúde Pública da Universidade de São Paulo, Universidade de São Paulo; 2002.

4. Chaves ITS, Grosseman S. O Internato Médico e Suas Perspectivas: estudo de caso com educadores e educandos. Rev. bras. educ. med. 2007; 31(3):212-22.

5. Werner JUHLW, Büchler MW. Acute pancreatitis. In: Cameron AM. Current surgical therapy. 8th ed. Philadelphia: Mosby; 2004. p. 459.

6. Fevereiro T, Caixas U, Pinto IV. Epidemiologia da pancreatite aguda na unidade de cuidados intensivos. Rev Port Med Int. 2000; 9(2):119-221.

7. Siegel S. Estatística não-paramétrica para as ciências do comportamento. São Paulo, McGraw-Hill 1975.

8. Komatsu RS, Padilha RQ, Caleman G. Interdisciplinaridade na educação médica: a experiência da Faculdade de Medicina de Marília (Famema). Rev. Adm. Pública. 2000; 34(6):81-93.

9. Tibério IFLC, Atta JA, Lichtenstein A. O aprendizado baseado em problemas - PBL. Rev Med. 2003 jan. Dez.; 82(14):78-80.
10. Ferreira RC, Tsuji H, Tonhom SFR. Aprendizagem Baseada em Problemas no Internato: há continuidade do processo de ensino e aprendizagem ativo? Rev. bras. educ. med. 2015; 39(2):276-285.

11. Ribeiro LRC. Aprendizado Baseado em Problemas. São Carlos: UFSCar: Fundação de Apoio Institucional; 2008.

12. Couto LB, Bestetti RB, Restini CB, Faria Jr M, Romão GS. Brazilian medical students' perceptions of expert versus non-exepert facilitators in a (non) problem-based learning environment. Med Educ Online. 2015 Apr 15; 20:26893.

13. Fukuda JK, Franzon O, Resende-Filho FO, Kruel NF, Ferri TA. Prognóstico dos casos de pancreatite aguda pelo escore de PANC 3. ABCD Arq Bras Cir Dig. 2013;26(2):133-5.

14. Augusto LGS, Câmara VM, Carneiro FF, Câncio J, Gouveia N. Environmental health: reflexions of the Brazilian Association of Post-Graduation in Collective Health-ABRASCO. Rev. bras. epidemiol. 2003;6(2):87-94.

15. Paulin LFRS, Poças RCG. A experiência da Universidade São Francisco com o internato médico de psiquiatria utilizando a metodologia da aprendizagem baseada em problemas. Rev psiquiatr RS. 2009;31(1):67-72.

\section{CONTRIBUIÇÃO DOS AUTORES}

Leonardo Castro Marinzeck: concepção e desenho da pesquisa, participou de todo o processo de elaboração do artigo.

Silvia Sidnéia da Silva: orientação da pesquisa, revisão de literatura, metodologia, interpretação dos resultados, revisão do artigo para encaminhamento à publicação.

Eduardo Stefaneli - revisão de literatura e revisão da análise dos dados.

Danilo Cesar Paschoalino - revisão de literatura e formatação do artigo.

Edilson Carlos Caritá: revisão do artigo e discussão do método, análise dos dados, elaboração inicial até a revisão do artigo para encaminhamento à publicação.

\section{CONFLITO DE INTERESSES}

Declaramos não haver conflitos de interesse

\section{ENDEREÇO PARA CORRESPONDÊNCIA}

Edilson Carlos Caritá

Programa de Mestrado Profissional em Saúde e Educação da Universidade de Ribeirão Preto

Av. Costábile Romano n. 2201 Bairro Ribeirânia

Ribeirão Preto, SP, Brasil

CEP 14096-990

Email: ecarita@unaerp.br 